PRePared For SUbMission to JINST

$15^{\text {Th }}$ Topical Seminar on Innovative Particle and Radiation Detectors,

14-17 OCTOBER 2019,

Siena, ItALY.

\title{
Modeling radiation damage in the pixel sensors of the ATLAS detector
}

\section{T. Lari, ${ }^{a}$ on behalf of the ATLAS Collaboration}

${ }^{a}$ INFN, Sezione di Milano, Milano, Italy

\section{E-mail: tommaso.lari@mi.infn.it}

\begin{abstract}
As the integrated luminosity delivered by the Large Hadron Collider increases, radiation damage effects are increasingly apparent in the response of the vertex detectors of the experiments. In this contribution, measurements of these effects are discussed for the Pixel Detector of the ATLAS experiment, which by the end of LHC Run 2 has received a fluence of up to $10^{15} \mathrm{n}_{\mathrm{eq}} \mathrm{cm}^{-2}$. Measurements of leakage current, Lorentz angle, and charge collection efficiency are discussed and compared to predictions from the Hamburg radiation damage model, TCAD based calculations, and a detailed simulation of the pixel sensor response which accounts also for the modifications to the electric field and charge collection efficiency induced by the radiation damage.
\end{abstract}

KeYwORDs: Solid State detectors, Radiation-hard detectors

${ }^{1}$ Copyright 2019 CERN for the benefit of the ATLAS Collaboration. CC-BY-4.0 license. 


\section{Contents}

1 The ATLAS Pixel Detector 1

2 Effects of Radiation damage 2

2.1 Leakage current 2

2.2 Effective doping concentration 3

2.3 Charge Collection Efficiency 4

3 The Radiation Damage Digitizer 6

4 Conclusions $\quad 8$

\section{The ATLAS Pixel Detector}

The Pixel Detector [1, 2] is the innermost component of the ATLAS experiment [3] which studies the collisions produced by the Large Hadron Collider [4]. It consists of four barrel layers and three end cap disks on each side, providing an average of four measurements for each charged particle with a high granularity and close to the interaction point. This coverage extends to $|\eta|<2.5^{1}$ and for the full azimuthal angle. The Pixel Detector gives ATLAS the capability to resolve even close-by tracks in the core of high transverse momentum jets, and allows excellent performance in the reconstruction of the primary vertices from proton-proton collisions and secondary vertices from the decay of B hadrons, charmed hadrons, and tau leptons, which is of critical importance for the flavour tagging capabilities of the experiment.

The innermost barrel layer, the Insertable B-layer (IBL) [2] was installed in 2014 before the start of LHC Run 2. It is located just $3.3 \mathrm{~cm}$ from the beam axis and uses planar oxygenated $n^{+}-\mathrm{in}-n$ sensors in the central region and $3 \mathrm{D} n^{+}-\mathrm{in}-p$ sensors in the forward region. The other barrel layers are located at radii of $5.05 \mathrm{~cm}, 8.85 \mathrm{~cm}$ and $12.55 \mathrm{~cm}$ and are called B-layer, Layer 1, and Layer 2 respectively; these layers as well as the disks have been operative since the start of Run 1. The size of the pixel cells is $50 \times 400 \mu \mathrm{m}^{2}$ in the outer three barrel layers and the disks, and $50 \times 250 \mu \mathrm{m}^{2}$ in the IBL, while the sensor thickness is $250 \mu \mathrm{m}$ in the outer layers and 200 (230) $\mu \mathrm{m}$ for the IBL planar (3D) sensors. A total of 92.4 million pixel cells cover a total active area of $1.88 \mathrm{~m}^{2}$.

The location of the Pixel Detector implies that it receives the highest fluence from the passage of the particles produced by the LHC collisions. The non ionizing energy loss (NIEL) of charged and neutral hadrons is particularly important for silicon radiation detectors, and it is expressed in

${ }^{1}$ ATLAS uses a right-handed coordinate system with is origin at the nominal interaction point (IP) in the centre of the detector and the $z$-axis coinciding with the axis of the beam pipe. The $x$-axis points from the IP towards the centre of the LHC ring, and the $y$-axis points upward. Cylindrical coordinates $(r, \phi)$ are used in the transverse plane, $\phi$ being the azimuthal angle around the $z$ axis. The pseudorapidty is defined in terms of the polar angle $\theta$ as $\eta=\ln \tan (\theta / 2)$. 
units of the fluence of $1 \mathrm{MeV}$ neutrons which would result in the same total NIEL. In these units, the IBL has received a total fluence of $10^{15} \mathrm{n}_{\mathrm{eq}} \mathrm{cm}^{-2}$ and the B-Layer a fluence of $5.510^{14} \mathrm{n}_{\mathrm{eq}} \mathrm{cm}^{-2}$. A comparable fluence will be added during LHC Run 3. After that, the Pixel Detector will be replaced and its successor the ITK Pixel tracker [5] would have to cope with an order of magnitude larger integrated luminosity. While the (current and future) pixel detectors have been designed to be radiation tolerant, their performance will still degrade over time. It is thus very important to measure and understand the effects of radiation damage on the detector response.

\section{Effects of Radiation damage}

The effects of radiation in a silicon sensor bulk are primarily caused by inelastic scattering of hadrons which displaces atoms from their lattice sites. The resulting lattice defects create energy levels in the band gap. At room temperature these also change with time due to the thermal motion of lattice defects, in a process called annealing. Annealing can be greatly accelerated, or suppressed, by higher and lower temperatures respectively. At a macroscopic level, there are three main consequences on the properties of a silicon sensor as a particle detector : the leakage current increases; the effective doping concentration changes with a net increase of acceptor-like states, and the signal is reduced due to trapping of electrons and holes by lattice defects. In the following, measurements of these three effects are presented. For the leakage current and effective doping concentration, measurements are compared to the prediction of the Hamburg model [12]. The modelling of the degradation of the signal charge is instead discussed in section 3 .

\subsection{Leakage current}

Leakage current is measured using the HVPP4 system during LHC Run 1 [6] and the module power supply system during LHC Run 2. The associated uncertainties on the measured values are 15.9\% and $11.2 \%$, respectively. The results are shown for the three outer barrel layers in Figure 1 where the measured values have been averaged for each layer and normalized to $0{ }^{\circ} \mathrm{C}$. The data span the entire Run 1 and Run 2 data taking periods, and are presented as a function of the integrated delivered luminosity since the beginning of Run 1; the total at the end of Run 2 in November 2018 being $191 \mathrm{fb}^{-1}$. The predictions from the Hamburg model are also reported in Figure 1, scaled to match the leakage current data.

The comparison of the measured leakage current with the predictions of the model allows the conversion factor between the integrated luminosity and the fluence for each pixel layer to be derived. In fact, this has been done separately for different bins of the longitudinal (along the beam direction $z$ ) position of the pixel detector modules inside each barrel layer, and is shown by the filled markers in Figure 2. For the three outer layers, no dependence of the fluence on the longitudinal position is observed, while for the IBL the conversion factor is larger for the central part of the detector. The fluence has also been predicted using Pythia8 [7, 8] to generate inelastic proton-proton interactions and either FLUKA $[9,10]$ or Geant4 [11] to simulate the particle interactions with the detector material. These predictions are indicated in Figure 2 as dotted and dashed lines. The predictions reproduce the lack of dependence on $z$ for the fluence in the outer layers, though the normalization is slightly smaller than that derived from the leakage current data. For the IBL, a 


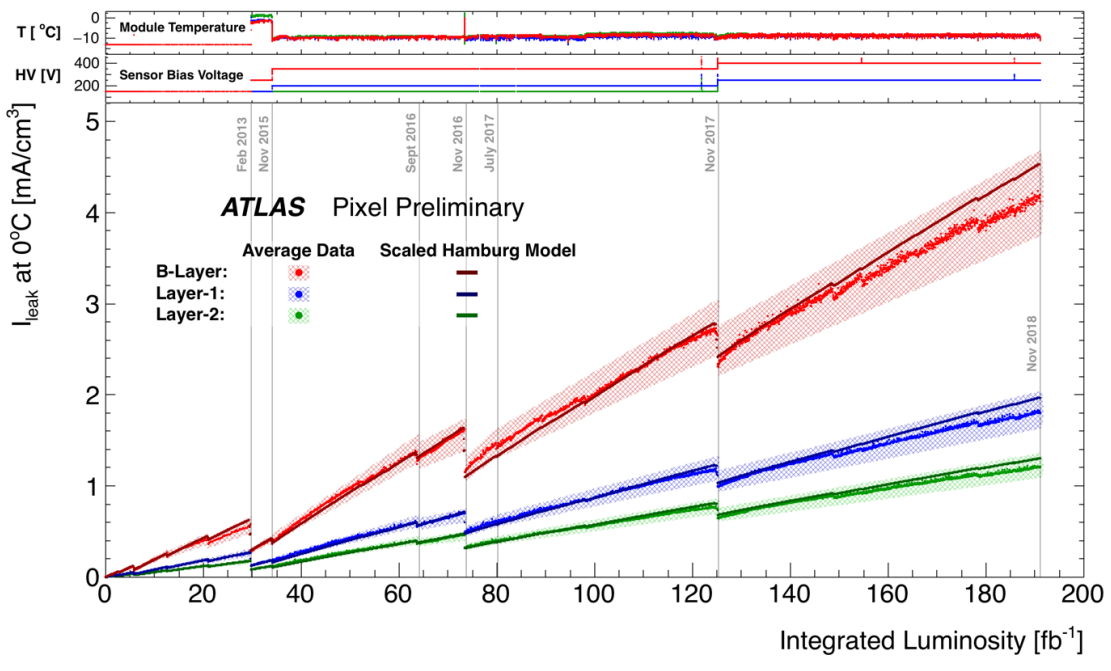

Figure 1. Average measured leakage current data of a representative set of modules of the ATLAS Pixel Barrel layers over the full period of operation. The Hamburg model predictions have been scaled to match the measured data. The leakage current data are normalized to $0{ }^{\circ} \mathrm{C}$; the average module sensor temperature is shown in the top panel. The average bias voltage is shown in the middle panel. Dates corresponding to the extended periods where the LHC beam was off, resulting in annealing of the sensors, are displayed within the lower panel for reference. From Ref. [13].

weak dependence of the fluence on $z$ is predicted, in contrast with the much stronger variation from the leakage current data.

The fluence can be also derived from the measurements of full depletion voltage presented in the next section. These measurements probe the relative fluence between $z$ bins and are normalized to the leakage current prediction at $z=0$ and indicated in Figure 2 as open markers. They support the strong dependence on $z$ extracted from leakage current data.

\subsection{Effective doping concentration}

In the Hamburg model, radiation damage removes donor states from n-type silicon and introduces defects which act as neutral or acceptor states. The net result is that the effective doping concentration initially decreases for n-type silicon; eventually the silicon bulk experiences type inversion and behaves as p-type silicon with an effective doping concentration which increases with time. This leads to an increase of the bias voltage which is required to fully deplete the silicon sensor.

Measurements of full depletion voltage were performed for the ATLAS Pixel detector using two different methods. Before type-inversion a measurement of the cross-talk between adjacent pixels gives a precise measurement of the full depletion voltage, since the p-n junction is located on the opposite side of the sensor and the pixels are isolated only after the full sensor thickness is depleted. After type inversion, the charge collection efficiency is measured as a function of the bias voltage in dedicated calibration runs. The charge increases rapidly with the bias voltage as long as the pixel sensor is partially depleted. The full depletion voltage is obtained by fitting with a linear function the rising and plateau regions of the measured data, and finding the bias voltage 


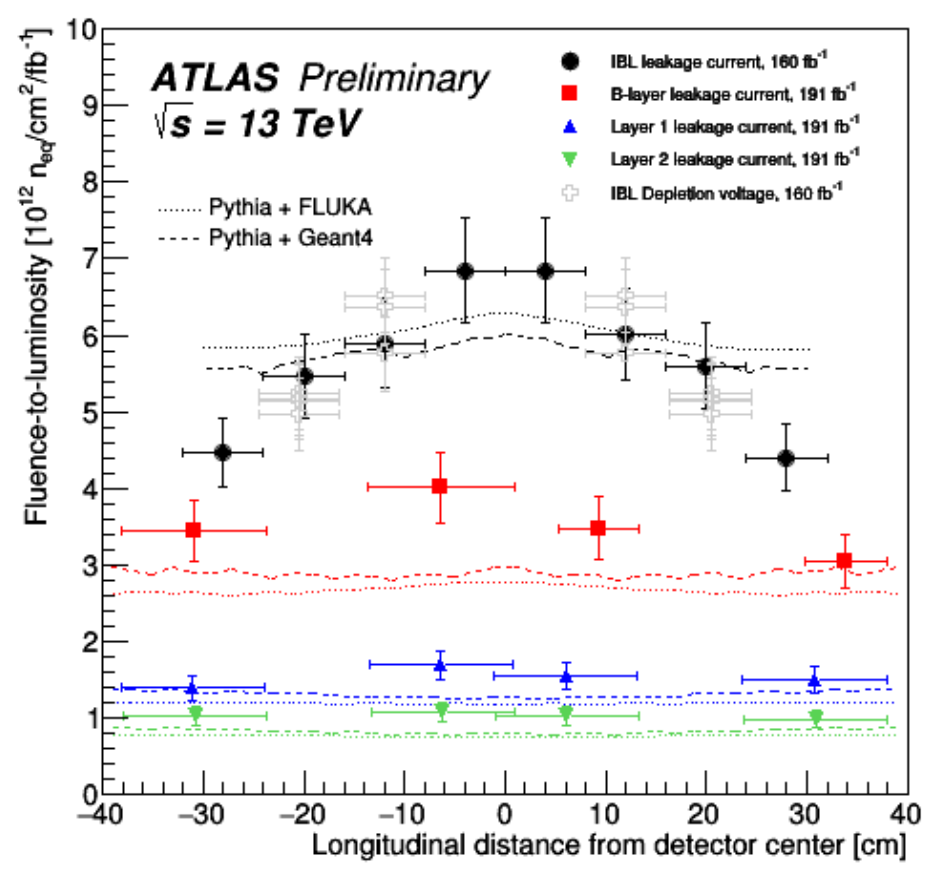

Figure 2. The extracted fluence-to-luminosity factors using measurements of leakage current (filled markers) and depletion voltage (empty markers). Predictions for the fluence are also reported for Pythia + Fluka (dotted lines) and Geant4 (dashed lines). From Ref. [14].

corresponding to the transition between the two regimes. The results are reported in Figure 3 for the B-layer. The data are the filled markers, while the predictions for the Hamburg model are the lines. The defect introduction rates used in the model have been fitted to the data. It can be seen that type inversion occurred at the end of Run 1. The full depletion voltage has increased ever since, and it is predicted to be around $300 \mathrm{~V}$ at the end of Run 2. This is smaller than the operating voltage of $400 \mathrm{~V}$ for the B-layer in 2018, and also well below the maximum operating bias voltage, which is $600 \mathrm{~V}$ for the outer three layers and $1000 \mathrm{~V}$ for IBL.

\subsection{Charge Collection Efficiency}

Another effect of the lattice defects introduced by radiation is the trapping of electron and holes before they reach the electrodes, resulting in a reduction of the electric signal on the pixel cells. Since the ATLAS Pixel Detector measures the collected charge using the time over threshold (ToT) of the signal, this can be directly measured. In Figure 4 the pixel cluster size and the charged particle average energy loss are shown as a function of the delivered integrated luminosity. The cluster size is defined as the number of adjacent pixel cells which collect a signal above the detection threshold; since the pixel matrix is two dimensional, this is measured separately in the two directions. The cluster size has some jumps when the operating conditions of the detector, in particular the value of the threshold and the bias voltage, have been changed. Between these jumps, it can be observed how the cluster size has a mild but definite decrease. This is due to the decrease in the collected charge which reduces the number of pixel cells whose signal is above the detection threshold. The effect is even more noticeable from the energy loss measurement, that for each reconstructed charged 


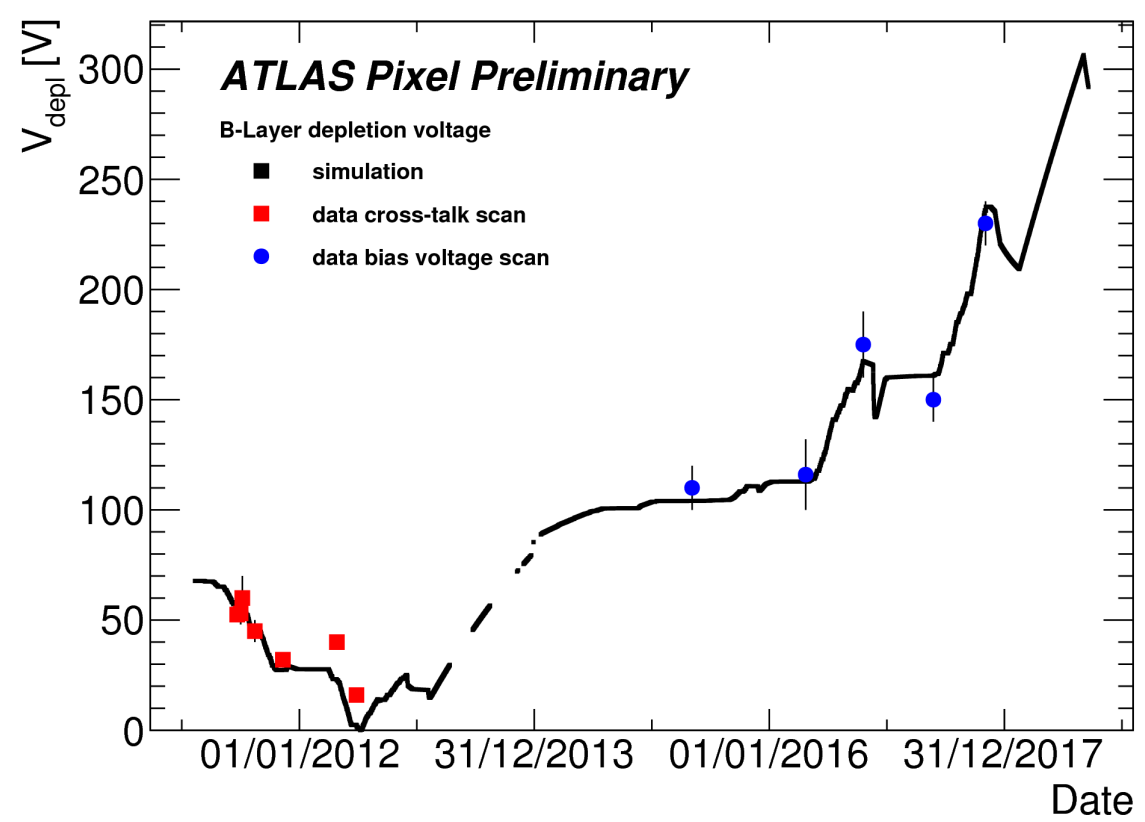

Figure 3. Measurements of the full depletion voltage using the cross-talk scan method (square points) and the bias voltage scan method (circular points) for the B-layer. Calculated values using the Hamburg model are also reported. From Ref. [15].

particle track is taken as the average from the charge (calibrated ToT) measurements in each of the pixel clusters contributing to the track. This steadily decreases with time.

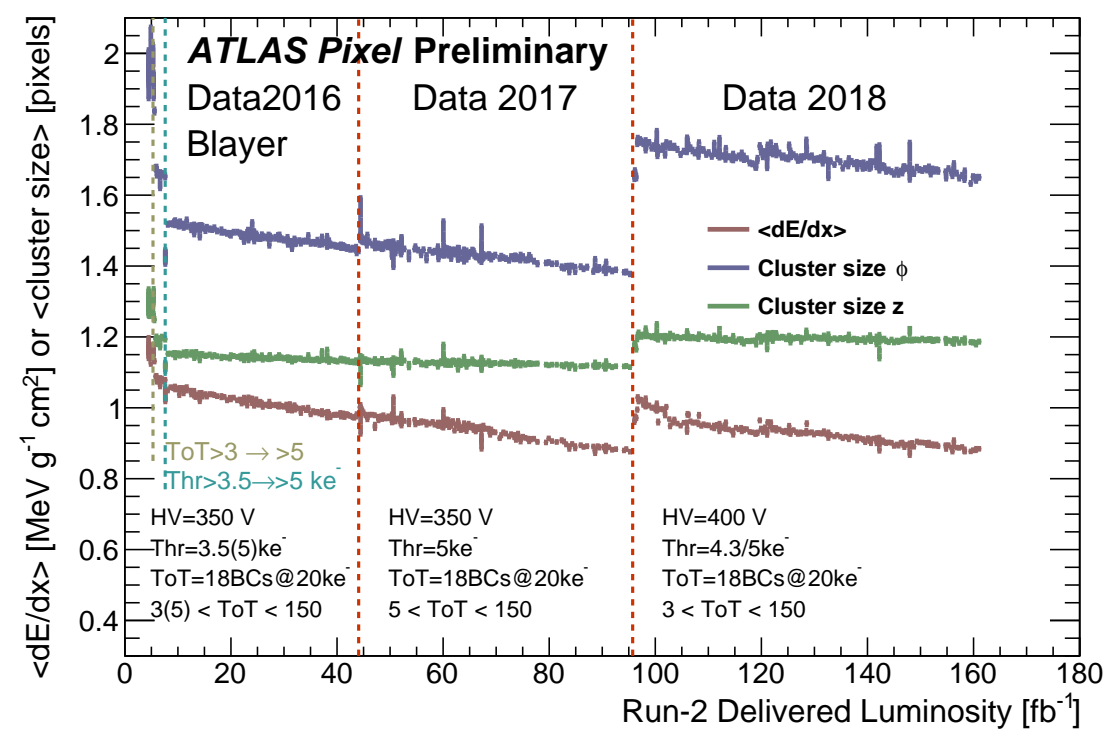

Figure 4. The dependence of the average cluster size and the measured $\mathrm{d} E / \mathrm{d} x$ on the delivered integrated luminosity. Red dotted lines mark the different data taking years. From Ref. [16] 
In the next section, a detailed simulation of the response of the pixel detector is described, which includes the effects of radiation damage.

\section{The Radiation Damage Digitizer}

Charged particles crossing a detector create electron-hole pairs, which then drift toward the electrodes under the effect of the electric and magnetic fields. The signal they induce on the collecting electrodes is converted to digital signals by the front end electronics.

In simulation, energy deposits are evaluated by Geant4 [11], a software package which evaluates the interaction of particles with the detector material, and produces a list of energy deposits in the sensitive detector elements, which are called hits. The calculation of the simulated digital signals from the hits is called digitization. A detailed model of digitization, including radiation damage effects, is implemented in the AllPix software tool [18]. The predictions of this tool have been used to validate the radiation damage model [17]. The model has also been implemented in the ATLAS common software Athena, in order to exploit the full geometry description of the ATLAS detector, and calculate the radiation damage effects on the ATLAS tracking, the performance in the reconstruction of physics objects (like jet flavour tagging), and the effects on the predicted background and signal observables in physics analyses.

First, the geometry of the sensor is loaded and Geant 4 is used to produce the hits in the sensor. Then all the other parameters are loaded : fluence, trapping time for electron and holes, the electric and magnetic field strength, and a number of lookup tables, describing the Ramo potential, the electric field, and the Lorentz angle as a function of the position inside the sensor. The electronhole pairs associated to the energy loss (one pair for each $3.6 \mathrm{eV}$ of energy loss) are drifted towards the electrodes using the information from the lookup tables. Based on the electric field strength, the time to reach the electrodes is calculated and compared to a random number describing the time before trapping; if the charge is trapped before reaching the electrodes, the signal induced on neighbour pixel cells is calculated using the Ramo potential.

The average electric field is given by the bias voltage divided by the sensor thickness. The distribution inside the sensor depends, however, on the initial doping concentration, the charged defect centres introduced by radiation, and the charge distribution generated by electron and hole trapping. A TCAD based software produces the electric field map inside a sensor using the Chiochia model [19] and the Perugia model [20] for the energy levels created by radiation in planar and 3D sensors respectively ${ }^{2}$. For large fluences, the trapped charge creates a double peak structure in the electric field, as shown in Figure 5.

The magnetic field affects the drift direction of electrons and holes; the Lorentz angle is defined as the angle between the drift direction and the electric field direction, and it is a function of the electric field strength. Since the electric field is not constant inside the sensor, electrons and holes follow curved trajectories. These are calculated during initialization and the average Lorentz angle is saved in a lookup table as a function of the initial and final position of the charge.

The trapping time is inversely proportional to the fluence $\Phi$ as $\tau=1 / \Phi \beta$. The proportionality constant $\beta$ has been measured [21-23] for electrons and holes and found to have a dependence on

\footnotetext{
${ }^{2}$ The Chiochia model only describes n-type doped silicon and can not be used for the p-type 3D sensors used in ATLAS.
} 


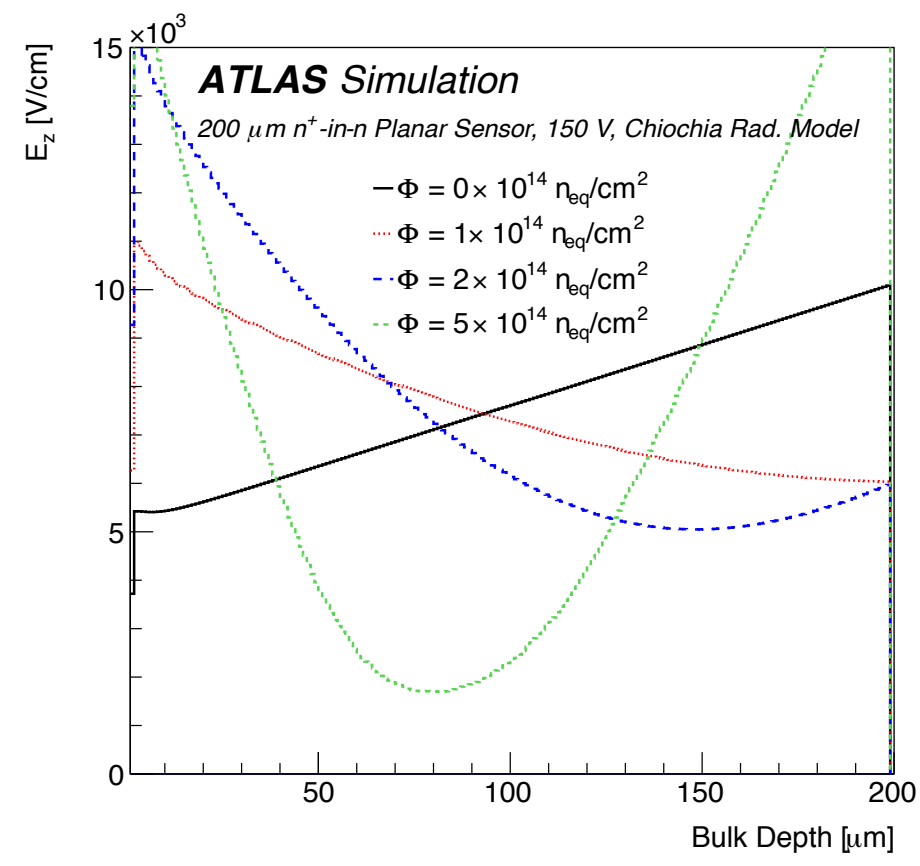

Figure 5. The simulated electric field magnitude across the sensor bulk depth for an ATLAS IBL sensor biased at 80 V. From Ref. [17]

the annealing time and on the type of radiation (charged or neutral hadrons). In the digitizer, an average of different measurements is used, with an uncertainty which covers the differences between measurements as well as the dependence on annealing time and irradiation type :

$$
\begin{aligned}
& \beta_{e}=(4.5 \pm 1.5) \times 10^{-16} \mathrm{~cm}^{2} / \mathrm{ns}, \\
& \beta_{h}=(6.5 \pm 1.5) \times 10^{-16} \mathrm{~cm}^{2} / \mathrm{ns} .
\end{aligned}
$$

where $\beta_{e}$ refer to the electron constant and $\beta_{h}$ to the hole constant.

The Ramo potential is used to calculate the charge induced on the electrodes by a charge $q$ moving from the position $\vec{x}_{\mathrm{i}}$ to the position $\vec{x}_{\mathrm{f}}$. Using the Shockley-Ramo theorem [24] this is $-q\left[\phi_{\mathrm{W}}\left(\vec{x}_{\mathrm{f}}\right)-\phi_{\mathrm{w}}\left(\vec{x}_{\mathrm{i}}\right)\right]$ where $\phi_{\mathrm{W}}$ is the Ramo potential, which depends only on the geometry of the electrodes and it is calculated in the initialization phase of the software.

In order to validate the digitizer and the radiation damage model it uses, the results from the simulation of a single pixel sensor in the simulation are compared to those measured in the ATLAS data. In figure 6 the results for charge collection efficiency (CCE) in the IBL are shown. The measured and simulated distributions of collected charge are fitted with a Landau distribution convoluted with a Gaussian to find the most probable value. The CCE is defined as this value normalized to that obtained for unirradiated sensors. The IBL bias voltage was increased repeatedly to cope with radiation damage : the detector was operated at $80 \mathrm{~V}$ in $2015,150 \mathrm{~V}$ in $2016,350 \mathrm{~V}$ in 2017 and $400 \mathrm{~V}$ in 2018. Thus, CCE values as a function of integrated luminosity were computed with the simulation for each of these values, and are reported as different curves in the figure. As can be seen, the value of CCE decreases with the integrated luminosity for each value of bias voltage, 
but this is compensated by the increases in bias voltage. Data are from the central $(|\eta|<0.6)$ modules of the IBL, and they show a fair agreement with the calculated values. At the end of Run 2 the CCE was about $70 \%$.

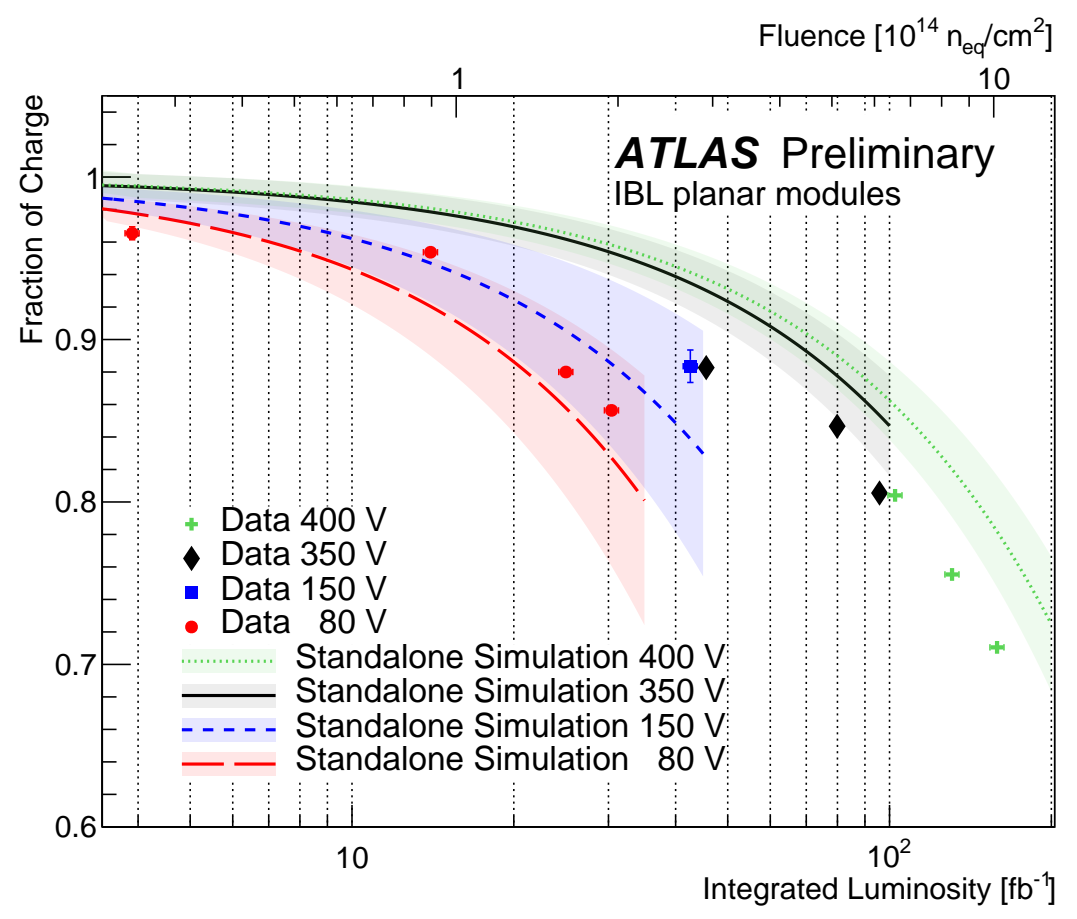

Figure 6. Charge collection efficiency as a function of integrated luminosity. From Ref. [25]

\section{Conclusions}

Effects of radiation damage in the ATLAS Pixel Detector are visible in the data collected at Run 2 of the LHC. Measurements of leakage current, full depletion voltage and charge collection efficiency have been presented and compared to predictions obtained with the Hamburg model and with a detailed implementation of the radiation damage effects in a pixel detector digitizer code.

\section{References}

[1] G. Aad et al., ATLAS Pixel Detector electronics and sensors, JINST 3 (2008) P07007.

[2] B. Abbott et al., Production and Integration of the ATLAS Insertable B-Layer, JINST 13(05) (2018) T05008

[3] ATLAS Collaboration, The ATLAS Experiment at the CERN Large Hadron Collider, JINST 3 (2008) S08003.

[4] L.Evans and P. Bryant (editors), LHC machine, JINST 3 (2008) S08001.

[5] ATLAS Collaboration, Technical Design Report for the ATLAS Inner Tracker Pixel Detector, http://cdsweb.cern.ch/record/2285585. 
[6] ATLAS Collaboration, A Leakage Current-based Measurement of the Radiation Damage in the ATLAS Pixel Detector, ATL-INDET-PUB-2014-004 (2014) http://cdsweb.cern.ch/record/1752122.

[7] T. Sjöstrand et al., Pythia 6.4 Physics and Manual, JHEP 05 (2006) 026, arXiv:hep-ph/0603175.

[8] T. Sjöstrand et al., An Introduction to Pythia 8.2, Comp. Phys. Comm. 191 (2015) 159, arXiv:1410.3012.

[9] G. Battistoni et al., The FLUKA code : Description and benchmarking, AIP Conf. Proc. 896 (2007) 31.

[10] A. Ferrari, P.R. Sala, A. Fasso and J. Ranft, FLUKA : A multi-particle transport code, CERN, 2005, http://cdsweb.cern.ch/record/898301.

[11] S. Agostinelli et al., Geant4 : a simulation toolkit, Nucl. Instr. and Meth. A 506 (2003) 250.

[12] M. Moll, Radiation damage in silicon particle detectors: microscopic defects and macroscopic properties, $\mathrm{PhD}$ thesis, Hamburg University (1999), https://www-library.thesis.de/cgi-bin/showprep.pl?desy-thesis99-040.

[13] ATLAS Collaboration, Measurements and Predictions of Pixel Detector Leakage Current, https://atlas.web.cern.ch/Atlas/GROUPS/PHYSICS/PLOTS/PIX-2018-008/.

[14] ATLAS Collaboration, Run 2 Leakage Current and Depletion Voltage Measurements for IBL, https://atlas.web.cern.ch/Atlas/GROUPS/PHYSICS/PLOTS/PIX-2019-001/.

[15] ATLAS Collaboration, Radiation damage in 2015-2017, https://atlas.web.cern.ch/Atlas/GROUPS/PHYSICS/PLOTS/PIX-2018-005/.

[16] ATLAS Collaboration, $d E d x$ and Cluster Size trends in Run-2 data, https://atlas.web.cern.ch/Atlas/GROUPS/PHYSICS/PLOTS/PIX-2018-011/.

[17] ATLAS Collaboration, Modelling radiation damage to pixel sensors in the ATLAS detector, JINST 14(06) (2019) P06012, arXiv:1905.03739.

[18] J. Idarraga, M. Benoit, Generic Geant4 implementation for pixel detectors, the AllPix Simulation Framework, (2006) [twiki.cern.ch:AllPix]

[19] V. Chiochia et al., A Double junction model of irradiated silicon pixel sensors for LHC, Nucl. Inst. and Meth. A 568 (2006) 51.

[20] F. Moscatelli et al., Combined Bulk and Surface Radiation Damage Effects at Very High Fluences in Silicon Detectors: Measurements and TCAD simulations, IEEE Trans. on Nucl. Science 63 (2016) 2716.

[21] O. Krasel et al., Measurement of trapping time constants in proton-irradiated silicon pad detectors, IEEE Trans. on Nucl. Science 51 (2004) 3055.

[22] G. Kramberger et al., Effective trapping time of electrons and holes in different silicon materials irradiated with neutrons, protons and pions, Nucl. Inst. and Meth. A 481 (2002) 297.

[23] G. Alimonti et al., A study of charge trapping in irradiated silicon with test beam data, (2003) ATL-INDET-2003-014.

[24] S. Ramo, Currents induced by Electron Motion, Proceedings of the IRE 27 (1939) 584, doi:10.1109/JRPROC.1939.228757.

[25] ATLAS Collaboration, Charge Collection Efficiency as a function of integrated luminosity, https://atlas.web.cern.ch/Atlas/GROUPS/PHYSICS/PLOTS/PIX-2017-004/. 